\title{
Nueva metodologia de evaluación del Holter basada en los sistemas dinámicos y la geometria fractal: confirmación de su aplicabilidad a nivel clínico
}

\author{
New methodology in Holter evaluation based \\ on dynamics systems and fractal geometry: \\ confirmation of its applicability at clinical level
}

\author{
Javier O. Rodríguez V. ${ }^{1,2}$, Signed E. Prieto B. ${ }^{1,2}$, Sandra C. Correa H., ${ }^{1,2}$, María Y. Soracipa M. ${ }^{1,2}$, Laura R. Méndez \\ P. ${ }^{1}$, Hebert J. Bernal C. ${ }^{1}$, Natalia C. Hoyos O. ${ }^{1}$, Laura P. Valero ${ }^{1}$, Alejandro Velasco R. ${ }^{1}$, Eduardo Bermudez ${ }^{3}$
}

Forma de citar: Rodríguez JO, Prieto SE, Correa SC, Soracipa MY, Méndez LR, Bernal HJ, et al. Nueva metodología del Holter basada en los sistemas dinámicos y la geometría fractal: confirmación de su aplicabilidad a nivel clínico. Rev Univ Ind Santander Salud. 2016; 48(1) 27-36. DOI: http://dx.doi.org/10.18273/revsal.v48n1-2016003 @e) (1) @

\section{RESUMEN}

Introducción: La teoría de sistemas dinámicos establece medidas cuantitativas de evolución de los sistemas mediante la construcción de atractores. Medidas de ocupación espacial de atractores cardiacos en el espacio fractal de Box Counting diferenciaron normalidad y enfermedad crónica de enfermedad aguda. Objetivo: Aplicar la metodología desarrollada para evaluar matemáticamente el estado cardiaco de Holter con diferentes patologías, confirmando la aplicabilidad de esta metodología para la detección de dinámicas agudas mediante medidas de concordancia estadística respecto al Gold Standard. Metodología: Se analizaron 170 Holter, incluyendo normales, crónicos y en estado agudo. Se construyeron simulaciones de la totalidad de la dinámica basada en número de latidos y frecuencia mínima y máxima cada hora durante 21 horas, para construir atractores en el espacio de fases. Se calculó la dimensión fractal de los atractores evaluando su ocupación espacial en el espacio de Box Counting, estableciendo cuáles corresponden a normalidad y enfermedad aguda de acuerdo con resultados matemáticos previos. Se comparó el diagnóstico matemático con el diagnóstico convencional del Holter, tomado como Gold Standard, estableciendo valores de sensibilidad, especificidad y coeficiente Kappa. Resultados: La dimensión fractal no logró evidenciar diferencias cuantitativas mientras que la metodología detectó en todos los casos dinámicas normales y en estado agudo independientemente de la patología, logrando valores de sensibilidad, especificidad, valor predictivo positivo y negativo de 100\%, y coeficiente Kappa de 1. Conclusiones: Se confirmó la capacidad de la metodología físico-matemática para detectar dinámicas agudas independientemente de la patología asociada, confirmando una auto-organización acausal de la dinámica del sistema cuya evaluación permite establecer medidas de aplicabilidad clínica.

Palabras Clave: Frecuencia cardiaca, diagnóstico, dinámicas no lineales, fractales.

1. Grupo Insight - Universidad Militar Nueva Granada. Bogotá D.C., Colombia

2. Centro de Investigaciones Clínica del Country. Bogotá D.C., Colombia

3. Universidad Surcolombiana. Neiva, Colombia

Correspondencia: Javier Rodríguez. Dirección: Cra. 79b\# 51-16 Sur, Interior 5, Apto. 102. Correo electrónico: grupoinsight2025@ yahoo.es. Teléfono: +514527541 . 


\section{ABSTRACT}

Introduction: Dynamic systems theory provides quantitative measures of evolution of systems by building attractors. Spatial occupation measures of cardiac attractors in fractal Box Counting space differentiated normality and chronic disease from acute illness. Objective: To apply the developed methodology to evaluate mathematically the cardiac status of Holter with different pathologies, confirming the applicability of this methodology for the detection of acute dynamic by statistical measures of agreement regarding the Gold Standard. Methodology: 170 Holter, including normal, chronic and in acute states were evaluated. Simulations were constructed the entire dynamic based on the number of beats and the minimum and maximum frequencies every hour for 21 hours, to build attractors in the phase space. The fractal dimension of attractors is calculated, evaluating the spatial occupation in the Box Counting space, establishing which corresponds to normal setting and acute disease in accordance with previous mathematical results. Mathematical diagnosis was compared with conventional diagnostic Holter, taken as the Gold Standard, setting sensitivity, specificity, positive and negative predictive value and Kappa coefficient. Results: The fractal dimension failed to show quantitative differences while the methodology detected in all cases normal dynamics and acute state independently of the disease, achieving sensitivity, specificity, positive and negative predictive value of $100 \%$ and a Kappa 1 . Conclusions: the ability of the physical-mathematical methodology to detect acute dynamic regardless of the associated pathology was confirmed, as well as an acausal self-organization of the system dynamics, which allows for assessment of clinical applicability measures.

Keywords: Heart rate, diagnosis, nonlinear dynamics, fractals.

\section{INTRODUCCIÓN}

Las enfermedades cardiovasculares son la primera causa de muerte en el mundo, y se estima que esta condición se mantendrá en el futuro próximo, siendo las formas isquémicas como el infarto agudo de miocardio (IAM) las que presentan mayor letalidad. La evaluación paraclínica convencional del estado de la dinámica cardiaca se hace mediante registros electrocardiográficos en el tiempo, como el test de Holter en donde se evalúan parámetros como el segmento ST y los intervalos R-R ${ }^{2}$.

El registro Holter es un examen no invasivo que brinda información como cambios del segmento ST -isquemia-, cambios en el intervalo QT, cambios en los intervalos R-R -variabilidad de la frecuencia cardiaca-, bradiarritmias y taquiarritmias, entre otras medidas $^{2}$. Sin embargo, el examen resalta la evaluación de eventos aislados tales como ectopias ventriculares o supraventriculares, y la evaluación de la totalidad de la información contenida en el Holter tiene un componente cualitativo, como puede observarse en las guías de práctica clínica ${ }^{3,4}$, lo que ha impedido desarrollar métodos de diagnóstico automatizados, por lo que se han buscado alternativas para mejorar su lectura, como el establecimiento de la variabilidad de la frecuencia cardiaca ${ }^{5-8}$. Debido a las limitaciones que tiene la forma actual de evaluación del test de Holter, se han desarrollado múltiples investigaciones desde la teoría de sistemas dinámicos, buscando establecer medidas de aplicabilidad clínica. La teoría de los sistemas dinámicos estudia el estado y la evolución de los sistemas mediante su representación gráfica en el espacio de fases. La representación gráfica de un sistema dinámico se hace posible mediante tres tipos de atractores caóticos, los cuales son de tipo cíclico, puntual y caótico9. Con la dimensión fractal es posible medir la irregularidad de los atractores de tipo caótico, al ser estos objetos de tipo irregular ${ }^{10}$.

La dinámica cardiaca es un sistema que va evolucionando en el tiempo, por lo que puede estudiarse con la teoría de los sistemas dinámicos. Dado que la dinámica cardiaca se considera impredecible y que resulta de gran inertes clínico poder establecer las características de su evolución, se han documentado múltiples medidas de sistemas biológicos útiles a nivel experimental y clínico mediante el uso de la teoría de los sistemas dinámicos, entre los que se encuentran la dinámica cardiaca fetal ${ }^{11,12} \mathrm{y}$ de adultos ${ }^{13}$. Goldberger et al., primero hallaron que la regularidad se asociaba a la anormalidad y la normalidad al comportamiento caótico, contrariamente a la concepción homeostática ${ }^{14}$. Posteriormente, establecieron que la enfermedad se caracteriza por presentar estados altamente regulares o irregulares, mientras que la normalidad se establece como un estado intermedio entre dichos extremos, lo que constituyó su segunda concepción de normalidad/ enfermedad ${ }^{15}$. Desde estas concepciones Huikuri et al., establecieron medidas predictoras de mortalidad que superan las medidas convencionales en pacientes con IAM y fracción de eyección menor al 35\%, a partir de medidas fractales de diferentes tipos, realizadas sobre el sistema dinámico ${ }^{16}$. 
El análisis de la variabilidad de la frecuencia cardiaca (HRV, por sus iniciales en inglés), es una de las medidas mayormente analizadas para la evaluación del ritmo cardiaco, sin embargo el análisis de la frecuencia cardiaca basado en teorías como la teoría del caos, la teoría de sistemas no lineales ${ }^{17-19}$ ha mostrado que el análisis clásico de HRV ha sido reevaluado como parámetro diagnóstico y predictor de la dinámica cardiaca. Se ha mostrado que la dinámica cardiaca presenta un comportamiento caótico o irregular ${ }^{20-22}$ lo que generó el desarrollo de una nueva concepción de salud - enfermedad ${ }^{15}$ aplicada a la fisiología cardiaca, en donde un trazado prácticamente plano y uno considerado aleatorio o altamente irregular, son asociados a la enfermedad, mientras que entre estos dos comportamientos se encuentra la normalidad. Se han desarrollado diferentes metodologías para el estudio de la dinámica de la frecuencia cardiaca ${ }^{23-26}$, sin embargo la aplicabilidad clínica de estas metodologías aún no está completamente establecida ${ }^{27,28}$.

Previo a la metodología aplicada en este trabajo, se desarrolló una metodología en la que se generaron todas las posibles arterias ${ }^{29}$ en el marco de un modelo experimental de restenosis, mostrando que las predicciones teóricas de la metodología abarcaban los resultados obtenidos experimentalmente; en este trabajo se desarrolló el concepto de espacio generalizado de Box-Counting, en el que es posible comparar geométricamente y de forma simultanea diferentes estructuras fractales, posteriormente este espacio fue utilizado en la metodología aplicada en este trabajo ${ }^{30}$, permitiendo diferenciar cuantitativamente normalidad y enfermedad crónica de enfermedad aguda, a partir de los espacios de ocupación de atractores cardiacos generados en dicho espacio.

Teniendo en cuenta este espacio se desarrolló una metodología de evaluación de la dinámica cardiaca con base en la teoría de los sistemas dinámicos, en la que se construyeron atractores caóticos de la frecuencia cardiaca, a partir de sus valores máximos y mínimos en el Holter durante cada hora; una vez obtenido el atractor para cada Holter se midió la evaluación de su ocupación espacial en el espacio de Box-Counting ${ }^{30}$ a partir de la superposición de dos rejillas necesarias para el cálculo de la dimensión fractal. Se calculó la dimensión fractal de Box-Counting, y se concluyó que esta medida no permite diferenciar las dinámicas normales de las enfermas, mientras que con los espacios de ocupación, medidos con la rejilla de 5 lat/min sí, pues esta rejilla permite una cuantificación más detallada de la ocupación espacial del atractor, lo que se refleja en las diferencias de magnitud establecidas entre los atractores, obteniéndose que una dinámica normal puede llegar a ocupar hasta 4 veces el espacio ocupado por una dinámica aguda, superando así las evaluaciones cualitativas del Holter.

Dicha metodología permitió establecer una autoorganización matemática del sistema dinámico cardiaco independiente de factores causales tales como edad, sexo u otras variables epidemiológicas, mediante la cual es posible diferenciar enfermedad aguda de enfermedad crónica y normalidad, evidenciando que los estados de normalidad son los que presentan una mayor ocupación espacial, mientras que estados agudos como el IAM presentan los menores valores de ocupación espacial ${ }^{30}$. Dicha metodología fue aplicada posteriormente a 150 Holter, incluyendo normales y enfermos, cuya contrastación con el Gold Standard permitió establecer valores de sensibilidad del 100\% en normalidad y patologías agudas, así como un coeficiente Kappa de 1, correspondiente al mayor nivel de concordancia posible ${ }^{31}$.

Dadas las implicaciones a nivel clínico de los hallazgos descritos, el propósito de este trabajo es realizar una aplicación de dicha metodología a 170 casos, con el fin de confirmar su aplicabilidad clínica para la detección de estados agudos independientemente de la patología observada, así como observar posibles asociaciones con la presencia de síncope.

\section{Definiciones}

Mapa de retardo: clase de atractor donde se simboliza gráficamente la dinámica del sistema, mediante la representación de pares consecutivos de la variable estudiada, en un espacio que puede ser bidimensional o de más dimensiones.

Fractal: Objeto irregular o irregularidad del mismo

Dimensión fractal: Medida numérica que evalúa la irregularidad de un objeto. En esta investigación se utilizará la definición de dimensión fractal de BoxCounting.

\section{Dimensión fractal de Box-Counting:}

$D=\frac{\log N_{1}\left(2^{-(K+1)}\right)-\operatorname{LogN}_{2}\left(2^{-K}\right)}{\log 2^{k+1}-\log 2^{k}}=\log _{2} \frac{N_{1}\left(2^{-(k+1)}\right)}{N_{2}\left(2^{-k}\right)}$ Ecuación 1

Donde:

N1: Número de espacios ocupados por el atractor en la cuadrícula de partición $\mathrm{K}$.

N2: Número de espacios ocupados por el atractor en la cuadrícula de partición $\mathrm{K}+1$ 
K: Grado de partición de la cuadrícula 1.

$\mathrm{K}+1$ : Grado de partición de la cuadrícula 2.

D: Dimensión fractal.

\section{Procedimiento}

Se realizó un muestreo de corte trasversal no probabilístico intencional, en el cual seleccionaron 170 Holter, provenientes de la base de datos del grupo Insight, de pacientes mayores de 20 años de edad, con diferentes patologías cardiacas, efectuados durante un mínimo de 21 horas, 40 de los cuales presentan un diagnóstico de normalidad desde los parámetros convencionales y 130 presentan algún tipo de patología cardiaca, de los cuales 40 presentaron patologías en estado agudo y los 90 restantes presentaron estados crónicos. Los diagnósticos desde los parámetros clínicos convencionales fueron revisados y validados por el especialista en cardiología.

Se tomaron las frecuencias cardiacas mínimas, máximas y el total de latidos cada hora durante mínimo 21 horas de cada uno de los Holter a estudiar. Se realizó una simulación de cada dinámica cardiaca de acuerdo con la metodología previamente desarrollada ${ }^{30,31}$. Se construyó el atractor caótico de cada una de las dinámicas en un mapa de retardo y se calculó su dimensión fractal. Para ello, se contabilizaron los espacios de ocupación de cada una de las escalas de 5 y 10 lat/min, empleadas en la aplicación de la metodología de Box-Counting, determinando los espacios de ocupación de cada atractor en las escalas evaluadas. La rejilla de 5 lat $/ \mathrm{min}$ se denominó kp mientras que la rejilla de 10 lat $/ \mathrm{min}$ se denominó kg.

De acuerdo con los valores obtenidos se estableció el diagnóstico físico matemático, evaluando los valores de ocupación del atractor encontrados, en comparación con los valores límites hallados para normalidad y enfermedad aguda, establecidos en el trabajo previo ${ }^{30}$.

\section{Análisis estadístico}

Posteriormente para los pacientes diagnosticados como normales y con patologías agudas, según el análisis convencional, se tomó dicho diagnóstico como Gold-Estándar, comparando este resultado con la metodología matemática calculando la especificidad, la sensibilidad, así como el Valor Predictivo Positivo y Negativo. Dichas medidas se realizaron a través de una clasificación binaria, donde los verdaderos positivos (VP) es el número de pacientes diagnosticados dentro de los límites de anormalidad y que se encuentran dentro de los valores matemáticos correspondientes al mismo diagnóstico, falsos positivos (FP) es el número de Holter que matemáticamente se comportan como estudios dentro de la anormalidad y cuyo diagnóstico clínico es normal, falsos negativos (FN) es el número de Holter diagnosticados clínicamente como anormales pero cuyos valores matemáticos se corresponden con pacientes normales $\mathrm{y}$, finalmente, verdaderos negativos (VN) definidos como el número de Holter diagnosticados clínicamente como normales y cuyos valores matemáticos también se corresponden con normalidad.

Posteriormente se evaluó la concordancia entre el diagnóstico obtenido a partir de la metodología físicomatemática y el diagnóstico clínico convencional mediante el cálculo del coeficiente Kappa a través de la siguiente fórmula:

$$
K=\frac{C o-C a}{T o-C a} \text { Ecuación } 2
$$

Donde:

Co: número de concordancias observadas, es decir, número de pacientes con el mismo diagnóstico de acuerdo con la nueva metodología propuesta y con el Gold Standard.

To: totalidad de observaciones.

$\mathrm{Ca}$ : Concordancias atribuibles al azar, que se calculan de acuerdo con la siguiente fórmula:

$$
C a=\left[\left(f_{1} x C_{1} / T o\right]+\left[\left(f_{2} x C_{2}\right) / T o\right] \text { Ecuación } 3\right.
$$

Donde $\mathrm{f}_{1}$ es el número de pacientes que presentan valores matemáticos dentro de los límites de normalidad, $\mathrm{C}_{1}$ es el número de pacientes diagnosticados clínicamente dentro de la normalidad, $\mathrm{f}_{2}$ es el número de pacientes que presentan valores matemáticos asociados a patología aguda aguda, $\mathrm{C}_{2}$ es el número de el número de pacientes diagnosticados clínicamente con patologías agudas y To es el número total de casos normales y con patologías agudas.

Según el artículo 11 de la resolución 008430 de 1993, del Ministerio de salud, el tipo de riesgo inherente a la investigación correspondería a una investigación de mínimo riesgo, dado que se realizan cálculos físicomatemáticos a partir de exámenes no invasivos prescritos como parte de los procedimientos habituales para los pacientes; cabe señalar que el anonimato y la integridad de los pacientes fue protegida. Se hace aclaración que se cumple con el artículo 13 de esta misma resolución ${ }^{32}$. El uso de consentimiento informado no es necesario debido a que la recolección de datos del estudio se haría en exámenes ya realizados, provenientes de un archivo de investigaciones previas del Grupo Insight. Se protegerá la privacidad del individuo, sujeto de investigación. 
Nueva metodología de evaluación del Holter basada en los sistemas dinámicos y la geometría fractal: confirmación de su aplicabilidad a nivel clínico

\section{RESULTADOS}

Las dimensiones fractales de los 170 Holter estudiados (Tabla 1) variaron entre 1,07 y 1,86 para los casos de normalidad y entre 1,16 y 2 para los Holter con alguna patología. Los espacios ocupados con la rejilla Kp presentaron valores entre 163 y 396 para normalidad y entre 21 y 228 para el grupo con diferentes patologías, de los cuales los Holter con enfermedad crónica presentaron valores entre 103 y 228 , en tanto que los que presentaron enfermedad aguda tuvieron valores entre 21 y 96 . Los espacios ocupados en la rejilla $\mathrm{Kg}$ presentaron valores entre 51 y 119 para normalidad y entre 6 y 72 para las diferentes patologías, encontrándose que los Holter con enfermedades crónicas presentaron valores entre 33 y 72 mientras que los Holter con enfermedad aguda presentaron valores entre 6 y 32 (Tabla 1 ).

Tabla 1. Diagnóstico establecido desde los parámetros convencionales, edad y medidas matemáticas realizadas para 25 de los Holter estudiados.

\begin{tabular}{|c|c|c|c|c|}
\hline No. Indicación / Conclusiones del estudio valorado convencionalmente & Edad & $\mathbf{K p}^{1}$ & $\mathbf{K g}^{2}$ & $\mathbf{D F}^{3}$ \\
\hline 1. Taquicardia. / Test dentro de límites normales & 27 & 382 & 111 & 1,78 \\
\hline 2. Dolor en el pecho. /Test dentro de límites normales & 56 & 361 & 101 & 1,83 \\
\hline 3. Arritmia./ Disminución de la variabilidad de la frecuencia cardíaca. & 74 & 193 & 54 & 1,83 \\
\hline 4. Control./ Extrasistolia ventricular frecuente & 54 & 187 & 60 & 1,64 \\
\hline 5. Taquicardia sinusalinapropiada. & 39 & 164 & 52 & 1,65 \\
\hline $\begin{array}{l}\text { Control. / Extrasístoles auriculares infrecuentes con una salva corta de taquicardia auricular no } \\
\text { sostenida }\end{array}$ & 60 & 153 & 43 & 1,83 \\
\hline 7. Postoperatorio de Ablación & 43 & 143 & 36 & 1,99 \\
\hline $\begin{array}{l}\text { Arritmia; extrasistoliasupraventricular frecuente con episodios de flutter auricular atípico o } \\
\text { 8. taquicardia auricular con respuesta ventricular rápida. Extrasistolia ventricular frecuente con } \\
\text { trigeminismo }\end{array}$ & 56 & 136 & 34 & 2 \\
\hline $\begin{array}{l}\text { 9. Palpitaciones / Ligera disminución de la variabilidad de la frecuencia cardíaca, 2. Leve tendencia } \\
\text { a taquicardia sinusal, } 3 \text { resto de estudio dentro de normalidad }\end{array}$ & 45 & 129 & 39 & 1,72 \\
\hline 10. Palpitaciones / Bloqueo de la conducción VIV & 49 & 121 & 36 & 1,74 \\
\hline $\begin{array}{l}\text { POP cambio valvular mitral y aórtico. Extrasistolia supraventricular y ventricular escasas con } \\
\text { algunas salvas de taquicardia auricular. Trastorno fijo de la repolarización }\end{array}$ & 21 & 113 & 34 & 1,73 \\
\hline 12. Dolor torácico / Disminución de la variabilidad de la frecuencia cardíaca. & 66 & 105 & 33 & 1,71 \\
\hline $\begin{array}{l}\text { 13. Sospecha de disfunción nodal; se concluyó fibrilación auricular severa, trastorno de conducción } \\
\text { intraventricular }\end{array}$ & 59 & 94 & 31 & 1,55 \\
\hline $\begin{array}{l}\text { Arritmia./Bradicardiasinusaldiurnasevera,bloqueoAV deprimergrado, extrasitoliasupraventricular } \\
\text { muy frecuente con episodios de bigeminismo. }\end{array}$ & 88 & 92 & 30 & 1,52 \\
\hline 15. Infarto Agudo de Miocardio & 72 & 68 & 19 & 1,83 \\
\hline 16. Disnea / Fibrilación auricular crónica. & 54 & 69 & 23 & 1,58 \\
\hline $\begin{array}{l}\text { 17. Control. / Fibrilación auricular, con respuesta ventricular media de } 89 \text { lpm. Extrasistolia ventricular } \\
\text { de manera muy infrecuente y aislada. }\end{array}$ & 71 & 65 & 23 & 1,49 \\
\hline 18. IAM & 64 & 62 & 16 & 1,95 \\
\hline 19. Fibrilación Auricular & 59 & 60 & 16 & 1,91 \\
\hline $\begin{array}{l}\text { 20. Disnea, dolor torácico / Infarto Agudo de Miocardio con elevación del ST anteroseptal KKII tipo } \\
\text { 1, Cardiopatía isquémica FEVI } 45 \% \text {, }\end{array}$ & 67 & 56 & 19 & 1,55 \\
\hline $\begin{array}{l}\text { 21. Dolor torácico. Infarto Agudo de Miocardio con elevación del ST cara anterior evolucionado } \\
\text { Killip I tipo 1, Enfermedad coronaria severa. }\end{array}$ & 45 & 47 & 18 & 1,38 \\
\hline 22. Fibrilación Auricular & 90 & 45 & 16 & 1,49 \\
\hline 23. Postoperatorio aislamiento pvi & 43 & 26 & 12 & 1,12 \\
\hline 24. Sincope, tormenta arrítmica y taquicardia ventricular no sostenida & 78 & 25 & 9 & 1,47 \\
\hline 25. Tormenta arrítmica & 62 & 23 & 9 & 1,35 \\
\hline
\end{tabular}

${ }^{1} \mathrm{Kp}$ : número de espacios ocupados en la rejilla de 5 lat $/ \mathrm{min}$.

${ }^{2} \mathrm{Kg}$ : número de espacios ocupados en la rejilla de 10 lat $/ \mathrm{min}$.

${ }^{3}$ DF: Dimensión Fractal. 
Se estableció que las medidas de dimensión fractal no permiten establecer diferencias entre estados de normalidad y enfermedad aguda, mientras que las medidas de ocupación espacial sí lo permiten (figura 1); los resultados evidencian que los pacientes que presentaron un diagnóstico clínico dentro de los límites normales presentan en todos los casos una ocupación de la rejilla Kp mayoro igual a163, en tanto que los Holter con patologías agudas presentan valores inferiores a 96, mientras que las diferentes enfermedades crónicas pueden presentar valores dentro del rango del grupo de Holter normales, o bien, presentar valores inferiores a los de normalidad, sin llegar a los de enfermedad aguda, confirmado los hallazgos del trabajo inicial ${ }^{30}$.

Se observó adicionalmente que 8 de los Holter evaluados presentaron síncope, el cual se asoció en todos los casos a un número de espacios de ocupación en la rejilla Kp igual o menor a 106, es decir, cercano o dentro del límite de enfermedad aguda, donde 7 de los 8 Holter presentaron valores inferiores a 97. A modo de ejemplo puede observarse el Holter No. 24 de la tabla 1, que presenta un valor Kp de 25. Este resultado puede ser indicador de que la metodología aplicada podría ser útil para la evaluación de la aparición de esta sintomatología en particular.

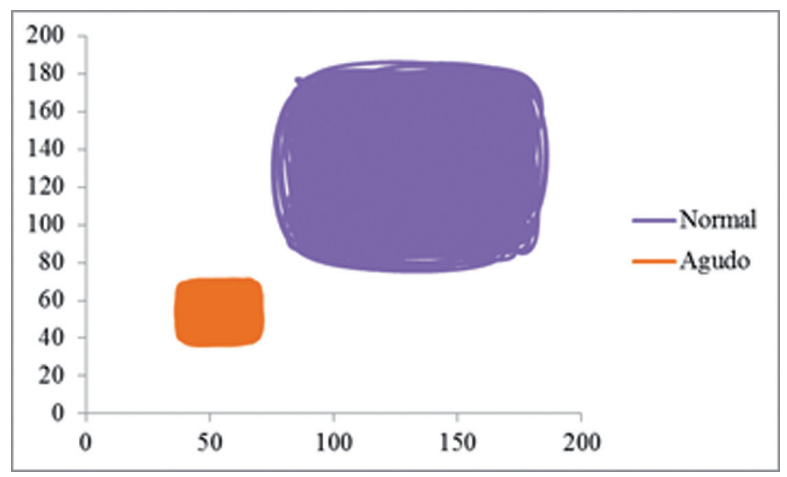

Figura 1. Gráficos de dos atractores caóticos en el mapa de retardo, la figura violeta corresponde a una dinámica cardiaca normal, mientras que la dinámica naranja corresponde a una dinámica con patología aguda como infarto agudo de miocardio. Obsérvese las diferencias en el espacio de ocupación entre los dos atractores.

\section{Resultados del análisis estadístico}

Mediante la comparación del diagnóstico físicomatemático basado en la ocupación espacial del atractor, respecto al diagnóstico convencional del Holter realizado por el cardiólogo experto, para los casos normales y con enfermedad aguda, se encontraron valores de sensibilidad y especificidad de $100 \%$. Se obtuvo un Valor Predictivo Positivo y Predictivo
Negativo de $100 \%$, así como un Coeficiente Kappa de 1. Estos valores evidencian que el método permite diferenciar normalidad de enfermedad aguda en todos los casos, confirmando la capacidad diagnóstica del método independientemente de la etiología del estado patológico.

\section{DISCUSIÓN}

Este es el primer trabajo en que, con base en una aplicación de la metodología de evaluación de la dinámica cardiaca fundamentado en la ocupación espacial de atractores en el espacio de Box Counting, a 170 Holter normales y con diferentes patologías, se obtiene una confirmación de su capacidad diagnóstica a nivel clínico para la detección de alteraciones de carácter agudo y su diferenciación con Holter normales. La contrastación del diagnóstico matemático respecto al diagnóstico convencional para los casos diagnosticados convencionalmente como normales y con patologías de carácter agudo mediante el establecimiento de medidas de sensibilidad, especificidad y coeficiente Kappa permitió determinar que se obtienen los valores de validez y concordancia más altos posibles, confirmando el carácter autoorganizado de la dinámica cardiaca, y el hecho de que la ocupación espacial en el espacio geométrico de Box Counting disminuye a medida que la dinámica se agudiza, lo que puede ser de utilidad para el seguimiento de un mismo paciente en el tiempo al permitir establecer cuantitativamente si la dinámica permanece estable, se dirige a valores característicos de normalidad o evoluciona a un estado de mayor gravedad.

Esta metodología simplifica la forma de evaluación convencional, que debe tener en cuenta múltiples parámetros, al lograr una evaluación cuantitativa basada en un solo valor. A diferencia de otros métodos para la construcción de atractores, que requieren de la recopilación de la secuencia total de frecuencias cardiacas para su determinación, en este caso al estar basada en una simulación realizada dentro de los rangos fisiológicos y clínicos hallados experimentalmente se demostró que se hace innecesario contar con la totalidad de frecuencias cardiacas para la obtención de la dinámica.

Se confirmó que las dimensiones fractales aisladas no permiten establecer diferencias diagnósticas, ya que sus valores se superponen al ser comparados entre diferentes tipos de pacientes. Este fenómeno ha sido observado en trabajos previos ${ }^{29,33-35}$, por lo cual ha sido necesario crear nuevas perspectivas mediante 
nuevos conceptos en cardiología y otras áreas, que han permitido establecer la diferenciación entre normalidad y enfermedad. En trabajos de monitoría cardiaca fetal, primero $^{11,12}$ se cuantificaron grados de complejidad fractales de monitorias normales en el centro, de enfermas con desaceleraciones de la frecuencia cardiaca y muy planas, en los extremos. También se cuantificaron con proporciones $\mathrm{S} / \mathrm{k}^{36}$ dinámicas cardiacas fetales en el centro de normalidad y en los extremos de normalidad. Ambos trabajos ya no son una concepción normalidad/ enfermedad sino cuantificaciones diagnósticas estrictas.

Gracias a la construcción de un espacio abstracto denominado espacio generalizado de Box Counting, donde se establecen comparaciones geométricas entre diferentes atractores al graficarlos simultáneamente en un mismo espacio, es posible establecer diferencias respecto a su ocupación espacial, lo cual ha permitido generalizaciones en varios fenómenos entre ellos, la dinámica cardiaca, como se puede observar en la metodología base para este estudio ${ }^{30}$, y la restenosis arterial ${ }^{29}$, en donde, para cada caso, se ha logrado establecer todas las posibilidades del fenómeno, mostrando que los fenómenos son acotados y finitos, estableciendo predicciones teóricas sobre lo que se consideraba caótico e impredecible. Este mismo espacio también permitió el desarrollo de una ley de los sistemas dinámicos caóticos cardiacos ${ }^{37-39}$.

En cardiología del adultos se ha cuantificado la normalidad y la enfermedad y la evolución entre las dos a partir de una ley exponencial mediante la cual se predicen todos los atractores cardiacos posibles ${ }^{37}$; esta ley se ha aplicado a pacientes con $\operatorname{arritmias}^{38} \mathrm{y}$ a pacientes con diferentes patologías, confirmando su capacidad diagnóstica ${ }^{39}$. Aplicando probabilidad y entropía a atractores caóticos se desarrollaron predicciones que diferencian normalidad de enfermedad crónica y aguda, así como la evolución entre cada estado ${ }^{40}$, metodología confirmada en un estudios ciegos aplicados a $300^{41}$, $450^{42}$ y $600^{43}$ casos normales y de pacientes con diferentes patologías cardiacas. Su utilidad también se ha confirmado en la predicción de procesos de agudización en la Unidad de Cuidados Coronarios ${ }^{44,45}$.

En el presente trabajo se comprueba para la metodología aplicada, que no son las dimensiones fractales las que permiten establecer la diferenciación entre salud y enfermedad para la dinámica cardiaca, sino los espacios de ocupación del atractor, lo que permite realizar mejores seguimientos dinámicos individuales. Los resultados obtenidos corroboran los resultados previos $^{30,31}$, que evidencian que la ocupación del espacio fractal permite diferenciar normalidad de enfermedad aguda. Se observó además que la indicación de síncope se asoció en todos los casos a valores en la rejilla $\mathrm{Kp}$ cercanos o inferiores a enfermedad aguda, señalando una posible asociación significativa a nivel clínico que debe ser analizada en estudios posteriores, pues podría llegar a ser de gran utilidad para la evaluación de este tipo de manifestación clínica específica.

Desde esta perspectiva físico-matemática se han logrado diagnósticos y predicciones objetivas y reproducibles en fenómenos como la diferenciación de estados de normalidad y anormalidad en eritrocitos ${ }^{46} \mathrm{o}$ arterias coronarias en el proceso de restenosis ${ }^{29}$, así como el diagnóstico de lesiones preneoplásicas y neoplásicas de cuello uterino ${ }^{47}$ y la predicción de linfocitos T CD4 en pacientes con HIV/SIDA a partir de información del cuadro hemático ${ }^{48}$. También se han desarrollado predicciones en inmunología ${ }^{49}$, biología molecular ${ }^{50}$ o salud pública, donde se desarrolló un método de predicción de brotes de malaria en 820 municipios de Colombia en períodos de tres semanas, con una exactitud del $99.86 \%^{51}$, así como predicciones de sepsis desde los sistemas dinámicos cardiacos en neonatos ${ }^{52}$. Desde esta perspectiva es posible que se logren generalizaciones de la totalidad de los fenómenos fisiológicos desde leyes físicas, estableciendo un fundamento físico para el estudio de la fisiología.

\section{AGRADECIMIENTOS}

Producto derivado del proyecto MED-1345, financiado por el fondo de Investigaciones de la Universidad Militar Nueva Granada. Bogotá, Colombia.

A la Universidad Militar Nueva Granada, por su apoyo a nuestras investigaciones, en especial al fondo de Investigaciones de la Universidad y a la Facultad de Medicina por su apoyo. Agradecemos de manera especial al Dr. Fernando Cantor, Vicerrector de Investigaciones, a la Dra. Martha Bahamón, Vicerrectora Académica, al Dr. Jorge Enrique Luque, decano de la Facultad de Medicina y al Teniente Coronel Médico Luis Antonio Castro Gómez, director del Centro de Investigaciones de la Facultad de Medicina por su contante apoyo y colaboración en nuestras investigaciones.

De igual forma al Centro de Investigaciones de la Clínica del Country por apoyo a nuestras investigaciones; a los Doctores Tito Tulio Roa, Director de Educación Médica, Jorge Ospina, Director Médico, Alfonso Correa, Director del Centro de Investigaciones, y a las Doctoras Adriana Lizbeth, epidemióloga, Sandra 
Rodríguez, enfermera, y Silvia Ortiz, Enfermera Jefe del Centro de Investigaciones.

\section{CONFLICTOS DE INTERÉS}

Los autores declaran no tener conflictos de interés.

\section{REFERENCIAS}

1. García M, García J. Prevalencia de factores de riesgo cardiovascular en jóvenes de una institución universitaria. Rev Salud pública. 2012; 14(5): 822830.

2. Pineda M, Matiz H, Rozo R. Enfermedad coronaria. Bogotá: Editorial Kimpres Ltda; 2002.

3. Motalescot G, Sechtem U, Achenbach S, Andreotti $\mathrm{F}$, Arden $\mathrm{Ch}$, et al. Guía práctica clínica de la ESC 2013 sobre diagnóstico y tratamiento de la cardiopatía isquémica estable. Rev Esp Cardiol. 2014; 67(2): 135.e1-e81.

4. American Heart Association \& European Society of Cardiology Guidelines. Heart rate variability Standards of measurement, physiological interpretation, and clinical use. Europ Heart J. 1996; 17: 354-381.

5. Chen WL, Kuo CD. Characteristics of Heart Rate Variability Can Predict Impending Septic Shock in Emergency Department Patients with Sepsis. Acad Emerg Med. 2007; 14(5): 392-397.

6. Chen WL, Chen JH, Huang CC, Kuo CD, Huang CI, Lee LS. Heart rate variability measures as predictors of in-hospital mortality in ED patients with sepsis. Am J Emerg Med. 2008; 26(4): 395401. DOI: 10.1016/j.ajem.2007.06.016.

7. Wu GQ, Arzeno NM, Shen LL, Tang DK, Zheng DA, Zhao NQ, et al. Chaotic Signatures of Heart Rate Variability and Its Power Spectrum in Health, Aging and Heart Failure. PLoS ONE. 2009; 4(2): e4323. DOI: 10.1371/journal.pone.0004323.

8. Braun C, Kowallik P, Freking A, Hadeler D, Kniffki K, Meesmann M. Demonstration of nonlinear components in heart rate variability of healthy persons Am. J. Physiol. 1998; 275: H1577-H1584.

9. Devaney R. A first course in chaotic dynamical systems theory and experiments. Reading Mass: Addison- Wesley; 1992.

10. Peitgen H. Strange attractors, the locus of chaos. En: Chaos and Fractals: New Frontiers of Science. New York: Springer-Verlag; 1992.

11. Rodríguez J, Prieto S, Ortiz L, Bautista A, Bernal P, Avilán N. Diagnóstico Matemático de la monitoria fetal aplicando la ley de Zipf-Mandelbrot. Rev Fac Med. Univ Nac Colomb. 2006; 54(2): 96-107.
12. Rodríguez J. Dynamical systems theory and ZipfMandelbrot Law applied to of a fetal monitoring diagnostic methodology. XVIII FIGO World Congress of Gynecology and Obstetrics. Kuala Lumpur, Malaysia. November 2006.

13. Higgins JP. Nonlinear systems in medicine. Yale J Biol Med. 2002; 75(5-6): 247-260.

14. Goldberger AL, Rigney DR, West BJ. Chaos and fractals in human physiology. Sci Am. 1990; 262(2): 42-49.

15. Goldberger AL, Amaral LA, Hausdorff JM, Ivanov PCh, Peng CK, Stanley HE. Fractal dynamics in physiology: alterations with disease and aging. Proc Natl Acad Sci USA. 2002; 99Suppl 1: 2466-2472.

16. Huikuri HV, Mäkikallio TH, Peng CK, Goldberger AL, Hintze U, Møller M. Fractal correlation properties of R-R interval dynamics and mortality in patients with depressed left ventricular function after and acute myocardial infarction. Circulation 2000; 101(1): 47-53.

17. Lombardi F. Chaos theory, heart rate variability, and arrhythmic mortality. Circulation. 2000; 101(1): 8-10.

18. Peng CK, Havlin S, Stanley HE, Goldberger AL. Quantification of scaling exponents and crossover phenomena in nonstationary heartbeat time series. Chaos. 1995; 5(1): 82-87.

19. Mäkikallio TH, Hoiber S, Kober L, Torp-Pedersen C, Peng CK, Goldberger AL, et al. Fractal analysis of heart rate dynamics as a predictor of mortality in patients with depressed left ventricular function after acute myocardial infarction. Am J Cardiol. 1999; 83(6): 836-839.

20. Garfinkel A, Spano ML, Ditto WL, Weiss JN. Controlling Cardiac Chaos. Science. 1992; 257(5074): 1230-1235.

21. Gough N. Fractals, chaos, and fetal heart rate. Lancet. 1992; 339: 182-183.

22. Goldberger AL. Is the normal heartbeat chaotic or homeostatic? News in Physiological Science. 1991; 6: 87-91.

23. Baumert M, Baier V, Voss A. Estimating the complexity of heart rate fluctuations - an approach based on compression entropy. Noise Lett. 2005; 4: L557-L563.

24. Beckers F, Verheyden B, Aubert AE. Aging and nonlinear heart rate control in a healthy population. Am. J. Physiol. Heart Circ. Physiol. 2006; 290: H2560-H2570.

25. Bär KJ, Boettger MK, Koschke M, Schulz S, Chokka P, Yeragani VK, et al. Non-linear complexity measures of heart rate variability in acute schizophrenia. Clin. Neurophysiol. 2007; 118: 2009-2015. 
26. Khoo MC. Modeling of autonomic control in sleep-disordered breathing. Cardiovasc. Eng. 2008; 8: 30-41.

27. Voss A, Schulz S, Schroeder R, Baumert M, Caminal P. Methods derived from nonlinear dynamics for analysing heart rate variability. Phil. Trans. R. Soc. 2009; 367A: 277-296.

28. Perkiömäki J, Mäkikallio TH, Huikuri HV. Fractal and Complexity Measures of Heart Rate Variability. Clin. Exp. Hypertens. 2005; 2(3): 149-158.

29. Rodríguez J, Prieto S, Correa C, Bernal P, Puerta G, Vitery $\mathrm{S}$, et al. Theoretical generalization of normal and sick coronary arteries with fractal dimensions and the arterial intrinsic mathematical harmony. BMC Med Phys 2010; 10: 1-6.

30. Rodríguez J, Prieto S, Avilán N, Correa C, Bernal $\mathrm{P}$, Ortiz L, et al. Nueva metodología física y matemática de evaluación del Holter. Rev Colomb Cardiol. 2008; 15: 50-54.

31. Rodríguez J, Prieto S, Bernal P, Soracipa Y, Salazar $\mathrm{G}$, Isaza $\mathrm{D}$, et al. Nueva metodología de ayuda diagnóstica de la dinámica geométrica cardiaca dinámica cardiaca caótica del Holter. Rev Acad Colomb Cienc. 2011; 35(134): 5-12.

32. Ministerio de salud. Resolución número 8430. Por la cual se establecen las normas científicas, técnicas y administrativas para la investigación en salud. Bogotá D.C.; 1993.

33. Rodríguez J, Prieto S, Correa C, Bernal P, Tapia D, Álvarez L, et al. Diagnóstico fractal de disfunción cardiaca severa. Dinámica fractal de la ramificación coronaria izquierda. Rev Colomb Cardiol. 2012; 19(5): 225-232.

34. Rodríguez J, Prieto S, Ortiz L, Ronderos M, Correa C. Diagnóstico Matemático de Ecocardiografías pediátricas con medidas de dimensión fractal evaluadas con Armonía Matemática Intrínseca. Rev Colomb Cardiol. 2010; 17: 79-86.

35. Rodríguez J, Prieto S, Correa C, Bernal P, Álvarez L, Forero G, et al. Diagnóstico fractal del ventriculograma cardiaco izquierdo. Geometría fractal del ventriculograma durante la dinámica cardiaca. Rev Colomb Cardiol. 2012; 19(1): 18-24.

36. Rodríguez J. Nuevo diagnóstico físico y matemático de la monitoria fetal: predicción de aplicación clínica. Momento Revista de Física. 2012; 44: 49-65.

37. Rodríguez J. Mathematical law of chaotic cardiac dynamics: Predictions for clinical application. J. Med. Med. Sci. 2011; 2(8): 1050-1059.

38. Rodríguez J, Narváez R, Prieto S, Correa C, Bernal P, Aguirre G, et al. The mathematical law of chaotic dynamics applied to cardiac arrhythmias.
J. Med. Med. Sci. 2013; 4(7): 291-300.

39. Rodríguez J, Correa C, Melo M, Domínguez, D, Prieto S, Cardona DM, et al. Chaotic cardiac law: Developing predictions of clinical application. J. Med. Med. Sci. 2013; 4(2): 79-84.

40. Rodríguez J. Entropía proporcional de los sistemas dinámicos cardiacos. Predicciones físicas y matemáticas de la dinámica cardiaca de aplicación clínica. Rev Col Cardiol. 2010; 17(3): 115-129.

41. Rodriguez J. Proportional Entropy applied to the Clinic Prediction of Cardiac Dynamics. Innovations in Cardiovascular Interventions, ICI meeting 2012.

42. Rodríguez J, Prieto S, Domínguez D, Melo M, Mendoza F, Correa C, et al. Mathematical-physical prediction of cardiac dynamics using the proportional entropy of dynamic systems. J Med Med Sci. 2013; 4(9): 370-381. DOI: http:/dx.doi.org/10.14303/ jmms.2013.080.

43. Rodríguez J, Prieto S, Correa C, Soracipa Y, Aguirre G, Méndez L. Proportional entropy applied to the clinical diagnostic of cardiac dynamic: blind study with 600 Holter. The 61st Annual Conference of the Israel Heart Society in association with The Israel Society of Cardiothoracic Surgery. 2014.

44. Rodriguez J. Proportional Entropy of the cardiac dynamics in CCU patients. 7th International Meeting of Acute Cardiac Care, Tel Aviv-Israel. 2011.

45. Rodríguez J, Prieto S, Bernal P, Izasa D, Salazar G, Correa C, et al. Entropía proporcional aplicada a la evolución de la dinámica cardiaca Predicciones de aplicación clínica. La emergencia de los enfoques de la complejidad en América Latina. Tomo I. Argentina: Comunidad del Pensamiento complejo. 2015; 247-264.

46. Correa C, Rodríguez J, Prieto S, Álvarez L, Ospino B, Munévar A, et al. Geometric diagnosis of erythrocyte morphophysiology. J. Med. Med. Sci. 2012; 3(11): 715-720.

47. Prieto S, Rodríguez J, Correa C, Soracipa Y. Diagnosis of cervical cells based on fractal and Euclidian geometrical measurements: Intrinsic Geometric Cellular Organization. BMC Medical Physics 2014, 14(2): 1-9.

48. Rodríguez J, Prieto S, Correa C, Pérez C, Mora J, Bravo J, et al. Predictions of CD4 lymphocytes' count in HIV patients from complete blood count. BMC Medical Physics. BMC Medical Physics. 2013; 13: 1-3.

49. Rodríguez J. Teoría de unión al HLA clase II teorías de Probabilidad Combinatoria y Entropía aplicadas a secuencias peptídicas. Inmunología. 2008; 27(4): 151-166. 
50. Rodríguez J, Bernal P, Prieto S, Correa C. Teoría de péptidos de alta unión de malaria al glóbulo rojo. Predicciones teóricas de nuevos péptidos de unión y mutaciones teóricas predictivas de aminoácidos críticos. Inmunología. 2010; 29(1): 7-19.

51. Rodríguez J. Método para la predicción de la dinámica temporal de la malaria en los municipios de Colombia. Rev Panam Salud Pública. 2010; 27(3): 211-218.

52. Rodríguez J, Prieto S, Flórez M, Alarcón C, López R, Aguirre G, et al. Physical-mathematical diagnosis of cardiac dynamic on neonatal sepsis: predictions of clinical application. J Med Med Sci. 2014; 5(5): 102-108. 